\section{Die am häufigsten gestellten Fragen zu TarMed}

Ich habe mich 1998 als Allgemeinarzt nach fast 5jähriger Weiterbildung - nicht nach FMH/WBO niedergelassen und habe den KVG-Ausweis erhalten, damit mir eine Konkordatsnummer zugewiesen wird.

Was wird mit meiner Abrechnung mit dem GRAT geschehen, wird es mir erlaubt sein, meine Leistungen wie bisher abzurechnen und, wenn ja, welche Leistungen?

Wenn ich bisher psychiatrische Positionen oder Interventionen abgerechnet habe, werde ich diese Leistungen weiterhin abrechnen können?

D. K. in L.

Im Rahmen der Übergangsbestimmungen werden Sie eingeladen, Ihr Tätigkeitsfeld zu definieren und sich einem bestimmten Facharzttitel zuzuordnen. Sie erhalten einen Ausweis, der Ihnen die Abrechnung in Analogie zu dem gewählten Facharzttitel erlaubt. Generell können Sie die Leistungen verrechnen, die Sie bereits erbracht haben; Sie haben diese in einer Selbstdeklaration festzuhalten.

Haben wir eine Vorstellung über den Taxpunktwert nach TarMed? Wann werden die exakten Werte bekannt sein?

A. G. in $L$.

Wissen wir noch nicht. Abhängig von den kantonalen Verhandlungen für den KVG-Bereich. Im UV/MV/ IV-Bereich wird die Festlegung bis Mitte Jahr erfolgen (schweizerischer Einheitstarif mit einheitlichem Taxpunktwert ganze Schweiz).

Bis jetzt habe ich die Traumarbeit meiner Gattin nach Pos. 769, delegierte Psychotherapie, nach Zürcher Tarif vom 1.7.1977 abgerechnet. Gibt es im TarMed eine ähnliche Position?

T. K. in R.

Delegierte Psychotherapie ist in der TarMed-Tarifstruktur enthalten. Ob Sie die Traumarbeit (traumhafte Arbeit? Arbeit an Träumen? Arbeit in Träumen? Arbeit im Träumen?) Ihrer Gattin verrechnen dürfen, bestimmt sich durch die kantonalen KVG-Verträge. Diesen kann in Sachen delegierte Psychotherapie ein gewisser Spannungsgehalt wie Unterhaltungswert auch in Zukunft gewiss nicht abgesprochen werden können.
Frage: Elektronische Rechnungsstellung.

1. Ist der Patient weiterhin Schuldner oder wurde nun das System umgestellt, so dass die Krankenkassen nun Tiers payant sind?

2. Wenn die Rechnung elektronisch direkt an die KK geht, erhält der Patient noch eine Rechnungskopie?

3. Falls der Patient weiterhin Schuldner ist, kann ich trotzdem mit den KK grössere Rechnungen ab Fr. 5000.- direkt abrechnen oder muss ich den Umweg über den Patienten gehen mit dem Risiko des Debitorenausfalles?

\section{B. A. in A.}

ad 1: TarMed funktioniert im "tiers garant» wie im "tiers payant»; im "tiers payant" bleibt der Patient weiterhin Schuldner des Arztes.

ad 2: Ja.

ad 3: Hängt vom Vertrag ab, der für Sie bzw. in Ihrem Kanton gilt: Diese Frage hat nichts mit TarMed zu tun.

Ich habe von unserem Medikamentenlieferanten gehört, dass das BAG droht, der Taxpunktwert des TarMed werde in den Kantonen mit Selbstdispensation deutlich tiefer angesetzt als in den anderen Kantonen (z.B. 60 Rp. statt Fr. 1.-). Für mich als Kinderpsychiaterin, die nur selten Medikamente abgibt, würde sich diese Regelung katastrophal auswirken, da ich in diesem Fall mit dem TarMed weniger verdienen würde als bisher (anstatt mehr, wie es den Psychiatern versprochen wurde; es war jeweils von einem Stundenhonorar wie im heutigen blauen Tarif die Rede). Ist an diesem Gerücht etwas Wahres, und wenn ja, was gedenkt die FMH dagegen zu unternehmen?

M. D. in $U$.

Der Taxpunktwert wird kantonal ausgehandelt, dies mit dem Ziel der sogenannten kostenneutralen Einführung. Für Patienten mit direkter Medikamentenabgabe durch den Arzt in relevantem Masse - der Kanton Aargau zählt zumindest auf dem Papier nicht $\mathrm{zu}$ diesen Kantonen - müsste einer Absenkung der Umsätze im Medikamentenbereich eine Anhebung des Taxpunktwertes für ärztliche Leistungen entsprechen - wovon theoretisch gerade Sie, die Sie ja keine Einkommenskomponente verlieren würden, nur zu gewinnen hätten. Unglücklicherweise liegen die Dinge etwas im Nebel, weil das BSV (und nicht das BAG) im Rahmen des KVG möglicherweise neue Regelungen für die direkte Medikamentenabgabe durch den Arzt/ die Ärztin erwägt. Die obigen grundsätzlichen Überlegungen zu Medikamentenabgabe und Taxpunktwert werden hierdurch nicht tangiert. 
1. Wann bekomme ich die Spezifikationen für die elektronische Rechnungsstellung bzw. für die Rechnungsgestaltung auf Papier?

2. Wann bekomme ich den TarMed-Tarif nicht als kompilierte HTML-Fassung, sondern in Form einer Datenbank (z.B. xBase .DBF)?

J. M. in B.

1. Die Spezifikationen für elektronische Abrechnung werden nach Massgabe der Verhandlungen eröffnet. Die grundsätzlichen Spezifikationen liegen vor. Fragen wie einheitliches Rechnungsformular sowie Detailspezifikationen stehen in Bearbeitung bzw. Verhandlung. Angaben können beim Sekretariat TarMed (Sekretariat TarMed, c/o Zentralstelle für Medizinaltarife UVG, Herrn Andreas Weissenburger, Postfach 4358, 6002 Luzern, Tel. 041 41952 59, Fax 04141957 04, E-mail: chsvlwes@ ibmmail.com) erfragt werden.

\section{Questions les plus fréquentes sur le TarMed}

Je me suis installé en tant que généraliste sans formation FMH en 1998. Après presque 5 ans de formation postgraduée, j'ai obtenu le certificat LaMal de formation postgraduée afin d'obtenir mon numéro de concordat auprès des caisses.

Que va-t-il se passer avec le GRAT concernant ma facturation, pourrais-je continuer à facturer mes prestations comme à ce jour et si oui lesquelles?

Si j'ai facturé des positions psychiatriques ou des interventions, est-ce que je pourrai continuer à les facturer?

D. K. $\dot{a} L$.

Dans le cadre des dispositions transitoires, vous serez invité à définir votre champ d'activité et à vous rattacher à un titre de spécialiste déterminé. Vous recevrez une attestation vous permettant de facturer en analogie avec le titre de spécialiste choisi. D'une manière générale, vous pourrez facturer les prestations que vous avez déjà fournies. Celles-ci devront être consignées dans une déclaration personnelle.
2. Den Anwendern ist die Tarifstruktur - wahlweise in Print oder elektronischer Form - so präsentiert, dass Sie Ihre Leistungen verrechnen können. Dies ist schon heute, Taxpunktwertfestlegung vorausgesetzt, der Fall. Die Eröffnung der zugrundeliegenden Datenbanken ist zur Erreichung dieser Zielsetzung nicht notwendig und deshalb auch nicht vorgesehen.

Avons-nous une idée de la valeur du point tarifaire pour TarMed? Quand est-ce que les valeurs exactes seront connues?

A. G. $\dot{a} L$.

Nous ne le savons pas encore. Cela dépendra des négociations cantonales pour le domaine de la LAMal. Dans le domaine de l'AA/AM/AI, elle sera déterminée d'ici à la fin juin (tarif et valeur du point unifiés pour l'ensemble du territoire suisse).

Jusqu'ici, j'ai facturé le travail de rêve de mon épouse selon la pos. 769, psychothérapie déléguée, du tarif

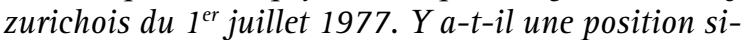
milaire dans le TarMed?

\section{T. K. $\dot{a} R$.}

La psychothérapie déléguée figure dans la structure tarifaire TarMed. La question de la facturation du travail de rêve de votre épouse (travail rêvé? Travail sur les rêves? Travail en rêvant?) est réglée par les conventions cantonales LAMal. En matière de psychothérapie déléguée, celles-ci ne sauraient manquer, à l'avenir également, de provoquer quelques tensions et débats sur la question. 
Question: facturation électronique

1. Le patient continue-t-il d'être débiteur ou le système a-t-il changé en faveur du tiers payant?

2. Si la facture est adressée directement par voie électronique aux caisses-maladie, le patient reçoit-il une copie de la facture?

3. Si le patient demeure débiteur, puis-je malgré tout facturer directement avec les caisses-maladie les factures d'un montant dépassant Fr. 5000.- ou dois-je passer par le patient, au risque d'une perte de débiteur?

$$
\text { B. A. } \dot{a} A \text {. }
$$

ad 1: Le TarMed fonctionne aussi bien avec le régime du tiers garant que du tiers payant. Dans le tiers payant, le patient demeure le débiteur du médecin.

ad 2: Oui.

ad 3: Cela dépend de la convention en vigueur dans votre canton: cette question n'a rien à voir avec le TarMed.

Mon fournisseur de médicaments m'a dit que l'OFSP menace d'abaisser sensiblement la valeur du point $d u$ TarMed dans les cantons qui pratiquent la propharmacie (Fr. 0,60 au lieu de Fr. 1.-). Pour moi, en tant que psychiatre d'enfants qui ne délivre que rarement des médicaments, cette réglementation s'avèrerait catastrophique, car avec le TarMed, je gagnerais moins qu'avant (au lieu de plus, comme cela a été promis aux psychiatres; il a toujours été question d'un salaire horaire comparable à celui du tarif bleu actuel). Ce bruit est-il fondé et, dans l'affirmative, que pense faire la FMH?

M. D. $\dot{a} U$.

La valeur du point est négociée sur le plan cantonal, l'objectif étant de maintenir ce que l'on appelle la neutralité des coûts lors de l'introduction du tarif. Pour les cantons où la distribution directe de médicaments par le médecin est très répandue - le canton d'Argovie ne fait pas partie de ces cantons, du moins sur le papier - une perte de revenu dans le domaine des médicaments devrait être compensée par une élévation de la valeur du point pour les prestations médicales, dont vous ne pourriez que profiter, puisque vous n'auriez pas à subir de perte sensible de revenu. Mais la situation n'est malheureusement pas encore éclaircie dans ce domaine parce que l'OFAS (et non l'OFSP) songe à mettre sur pied, dans le cadre de la LAMal, de nouvelles réglementations concernant la dispensation des médicaments par le médecin. Cela ne change rien, cependant, aux réflexions de fond évoquées ci-dessus sur la propharmacie et la valeur du point.
1. Quand pourrai-je obtenir les spécifications pour la facturation électronique ou les modalités de facturation sur papier?

2. Quand pourrai-je obtenir le tarif TarMed, non pas en version HTML compilée, mais sous la forme d'une banque de données (p. ex. xBase.DBF)?

J. M. $\dot{a} B$.

1. Les spécifications pour la facturation électronique s'aligneront sur les négociations. Les spécifications de base sont prêtes. Les questions telles que les formules de factures unifiées ou les détails de la spécification sont en cours d'élaboration ou de négociations. Pour plus de renseignements, vous pouvez vous adresser au secrétariat TarMed (c/o Service central des tarifs médicaux LAA, M. Andreas Weissenburger, case postale 4358, 6002 Lucerne, tél. 04141952 59, fax 04141957 04, E-Mail: chsvlwes@ibmmail.com).

2. Pour l'utilisateur, la structure tarifaire TarMed sur papier ou sous forme électronique - est présentée de manière à pouvoir facturer ses prestations. C'est déjà le cas aujourd'hui, hormis la détermination de la valeur du point. Pour atteindre cet objectif, l'accès aux banques de données de base n'est pas nécessaire et n'est donc pas prévu. 